\title{
NOTÍCIAS CULTURAIS.
}

\section{Seminário internacional de Direito Romano.}

\author{
A Redação.
}

Acontecimento marcante nos anais da Romanística internacional foi o Seminário Internacional de Direito Romano, realizado em Perugia (Itália), de 11 a 14 de outubro de 1971.

Ao Congresso, organizado pelo professor Mário DE Dominicis, diretor da Faculdade de Direito da Universidade de Perugia, compareceram ilustres juristas e mestres internacionais. Entre eles, o professor Alexandre Augusto DE CAStro CorrêAa, desta Faculdade, que apresentou a tese: Notas Sobre o Abuso do Direito no Direito Romano Clássico, debatida e aprovada na sessão sob a presidência do professor Jean Gaudemet da Universidade de Paris. Essa comunicação, redigida em francês, é publicada na secção de doutrina, deste fascículo.

Para melhor informar, publica-se, também a seguir, no original, o relatório elaborado pelo professor MÁRIO DE Dominicis.

Seminário Internazionale Romanistico. (Perugia 11-14 ottobre 1971)

II Seminario romanistico sorto per iniziativa dell'Istituto di Storia del diritto, sino dall' anno 1967-68, dopo il successo della XXII Sessione perugina della 'Société internationale F De Visscher pour l' étude des droits de l'antiquité' (S.I.D.A.), ha voluto promuovere un incontro tra i maggiori cattedratici del Sudamerica ed i piú illustri Maestri delle Uni- 
versità d' Europa, in concomitanza con la degna monumentale sistemazione della tomba di Bartolo da Sassoferrato nella trecentesca basilica di S. Francesco al Prato. L' importante manifestazione, durante la quale sono stati trattati temi di libera scelta, ma tutti di grande interesse scientifico, era stata preannunciata lo scorso anno dallo stesso suo anímatore, prof. Mario de Dominicis, al primo congresso sudamericano romanistico, nel senso piú lato della parola, svoltosi a Rio de Janeiro, sotto l'alto patronato del Presidente della Republica del Brasile.

I lavori sono stati aperti nell'aula magna dell' Università, presenti le massime Autorità cittadine e regionali, vari parlamentari dell' Umbria, tra cui gli onorevoli Lodovico Maschiella, Stefano Menicacci, Luciano Radi, Giuseppe Salari, Giorgio Spitella, gli ambasciatori di alcuni Stati del Sudamerica ed un folto gruppo di insigni Maestri di diritto romano, autorevoli rappresentanti di Paesi occidentali e socialisti, europei e d'oltre mare. Si é peraltro trattato, va subito messo in evidenza, di un incontro volutamente ristretto ai massimi esponenti della scienza romanistica e dei diritti dell' antichità, un po' di tutti i Continenti, ciò che ha conferito al convegno una qualificazione scientifica del tutto eccezionale.

Telegrammi di adesione e di compiacimento per l' iniziativa sono stati inviati dal Presidente del Senato, dal Ministro della Pubblica Istruzione, dal vice Presidente del Consiglio dei Ministri, on. prof. Francesco de Matino, egli stesso illustre cattedratico di Storia del diritto romano, da alte personalità del mondo della cultura d'Europa e d'America. Né va passato sotto silenzio il significativo messaggio del Presidente della Republica del Senegal, Leopold Sedar Senghor, nell'occasione rappresentato all'Università di Bartolo dal proprio ambasciatore a Roma. L'insigne Capo di Stato, uno dei più autorevoli ed illuminati dei nuovi Paesi africani, autore, tra l'altro, di un lavoro di larghissima noto- 
rietà sulla 'negritudine', ha voluto sottolineare il ruolo preminente che il diritto romano tuttora riveste nei piani di studio per il conseguimento della laurea e del dottorato nella giovane Università di Dakar, che si fregia del motto 'Lux mea Lex' I giuristi senegalesi - cosi conclude il messaggio del Presidente SEnghor - si sono formati alla scuola dell' antico diritto di Roma, per cui l'incontro internazionale romanistico perugino riveste ed assume per il suo Paese, un' importanza e un interesse particolare.

Nella seduta inaugurale, dopo un indirizzo di saluto dell'organizzatore del Seminario, prof. Mario DE Dominicis e brevi parole del Magnifico Rettore on. prof. Giuseppe ERMINI cui, nella circostanza é stata consegnata un'artistica medaglia d'oro con l'effigie di Bartolo - espressamente coniata - per le sua alte benemerenze di Maestro e Rettore dell'antico 'Studium Generale', ha preso la parola il prof. Robert Feenstra, dell'Università di Leiden.

Richiamandosi ai lavori del Congresso su Bartolo, svoltosi a Perugia nel 1959 - e specialmente al discorso introduttivo pronunciato in quell'ocasione dal compianto prof. F Calasso - l'oratore, dopo una breve panoramica sulla figura e l'opera di BARTolo, ha sottolineato la sua grande influenza nel secolo XIV ed oltre e le reazioni provocate specie nel periodo dell' umanesimo giuridico. Molti romanisti moderni insistono sulla utilizzazione di un metodo strettamente storico che hanno chiamato neo-umanista ma i metodi di studio oggi seguiti non sempre sono proprio e del tutto storici, come si pretenderebbe. Di fatto essi, continuano ad adottare nozioni moderne che ritengono essere le stesse dell' antico diritto romano. Queste nozioni peraltro hanno una loro propria storia, non soltanto nel periodo precedente alla codificazione giustinianea, ma anche e soprattutto nel medioevo e nei tempi moderni. Ocorre dunque, secondo il prof. Feenstra, studiare questo processo storico, sia per meglio 
comprendere le nozioni del diritto attuale, sia per evitare una falsa interpretazione dello stesso diritto romano.

Ha ricordato, a titolo di esempio, la nozione del 'dominium', precisando che i romanisti moderni si servono sempre della definizione datane da BARToLo, senza peraltro studiarla nel contesto in cui il sommo giurista ebbe ad elaborarla e senza considerarne l'evoluzione ulteriore, ciò che li porta ad attribuire ai giuristi romani lo stesso concetto della proprietà che si ritrova nelle codificazioni moderne. BARTolo e la sua scuola - ha concluso l'insigne studioso olandese - segnano una tappa memorabile nello sviluppo delle nozioni contenute nei testi, sempre fondamentali, del diritto romano.

Subito dopo i congressisti si sono recati nella basilica di S. Francesco al Prato per deporre una corona di alloro, con un nastro recante $\mathrm{i}$ colori del l'Università, sulla tomba del grande giureconsulto, di recente degnamente sistemata nella capella ex-Baldeschi. L'opera di restauro, di particolare effetto e finezza architettonica, pur nella sua semplicità, é dovuta alla lodevole iniziativa del prof. OTTORINo GURRIERI, cui va la gratitudine del mondo romanistico. Nell'occasione egli ha pure curato la stampa, in bella veste tipografica, di una dotta c pregevole memoria storica - omaggio della brigata perugina degli amici dell'arte ai partecipanti al S.I.R.P si legge nel frontispizio - sul monumento funebre del Maestro, dal titolo 'Bartholus hic iacet', le parole che grandeggiano all' esterno del sarcofago contenente i suoi Resti e suonano quasi come un monito sulla caducità delle cose umane.

A conclusione della mattinata inaugurale l'Amministrazione provinciale di Perugia offriva un signorile cocktail nella suggestiva Rocca Paolina. Il Seminario si é articolato in cinque sedute, di cui due tenute, rispettivamente, nella sala maggiore del palazzo Ancaiani di Spoleto, sede del centro italiano di studi sull'alto medioevo ed a Todi, nel salone delle conferenze dell'Accademia Tudertina. Il Comitato direttivo del S.I.R.P ha voluto, in tal modo, rendere omaggio 
a queste città che conservano due insigni documenti di diritto romano la 'lex luci Spoletini' e la 'lex Tudertina'. Alla loro presidenza si sono succeduti i professori: Hans Julius WolfF dell'Univ. di Freiburg; Joseph A. C. Thomas, dell'Univ. di Londra; Felix Wubbe dell'Univ. di Friburg; Dragomir Stoicevic dell'Univ. di Belgrado; Jean Gaudemet dell'Univ. di Parigi.

Dagli organizzatori é stato di proposito limitato il numero delle comunicazioni, per lasciare il maggior tempo possibili agli interventi ed alle discussioni. Ricorderemo, nell'ordine, $\mathrm{i}$ soli titoli delle relazioni svolte, in attesa della publicazione degli Atti che, dandoci un consuntivo preciso ed esauriente, potrà permettere di aprezzarne tutto il valore scientifico: Robert Feenstra (Leiden), Bartole et la Science du droit romain; Danilo Segoloni (Perugia), Bartolo e S. Francesco al Prato in Perugia; Aristide Theodorides (Bruxelles), Considérations sur le testament dans l'Egipte pharaonique; Ugo Brasiello (Roma), 'Plura crimina ex eodem facto'; MARio de Dominicis (Perugia), Cth. 16, 2, 30 (Sur les assemblées provinciales dans l'Afrique romaine); Gaetano Sciascia (Roma), Il senatoconsulto Planciano sui figli nati dopo il divorzio; Alfredo Mordechai Rabello (Jerusalem), II 'ius occidendi iure patris della lex Julia de de adulteriis e la vitae necisque potestas del pater familias); Carlo Castello (Genova), Sui rapporti tra 'dominus' e 'villicus' desunti dal 'De Agricoltura' di Catone; EmILE Szlechter (Paris), 'Locatio operarum' en droit romain et en droit babylonien; Arnaldo Biscardr (Milano), Un remoto precedente storico del prontuario bizantino 'de actionibus'; Alexandre Augusto Corkêa (São Paulo, Brésil), La doctrire de l'abus du droit en droit romain classique.

Molti e vivaci gli interventi - hanno animato le varie sedute, conferendo loro il vero carattere di seminario ad un alto e raro livello scientifico - specie da parte dei professori: Roberto Andreotti (Parma); Ayiter Kudret (Ankara); 
Gian Gualberto Archi (Firenze); Alexandre Augusto Corrêa (São Paulo, Brésil); Jean Gaudemet (Paris); Cesare Sanfilippo (Catania); Peter Stein (Cambridge); Emile Szlechter (Paris); Aristide Theodorides (Bruxelles); Joserh A. C. Thomas (Londra); Edoardo VolterRA (Roma); JaIme Williams (Santiago de Chile); Hans Julius WolfF (Freiburg); Felix Wubbe (Fribourg) ed altri.

Nella seduta di chiusura, svoltasi sotto la presidenza del prof. J. Gaudemet, il prof. M. DE Dominicis annunciava la sua iniziativa, d'intesa con il Magnifico Rettore dell'Ateneo on. prof. G. ErminI e con le autorità regionali, di promuoveve la costituzione di un 'Centro di studi costantiniani e del basso impero 'nel vecchio municipio romano di Hispellum, il cui nome é legato al celebre rescritto imperiale del 333-335, che può considerarsi la più antica carta statutaria dell'odierna autonoma Regione umbra. Il prof. Gaudemet, nel compiacersi vivamente per l'iniziativa, cui augurava, anche a nome di tutti gli studiosi, il migliore meritato successo, dava atto allo stesso prof. DE Dominicis della perfetta riuscita della manifestazione, lo ringraziava per le calorose accoglianze ricevute e, tra gli applausi dei presenti, dichiarava chiusi i lavori del S.I.R.P 1971.

L'incontro perugino non solo ha offerto l'occasione di poter conoscere i risultati scientifici più attuali, su temi di particolare interesse, raggiunti da personalità insigni della scienza romanistica, ma anche di fare ammirare le impareggiabili bellezze della natura e dell'arte di una tra le più belle e caratteristiche regioni d'Italia. Sono state visitate, sotto la dotta guida di esperti del luogo, i centri di Spoleto, Spello, Todi e infine Deruta, nota anche per le sue maioliche, di cui con gentile pensiero, l'Amministrazione civica ha voluto donare un artistico esemplare a tutti i Congressisti. 
Veramente ricca e generosa l'ospitalità umbra, sia nel capoluogo che nelle altre città della regione. Una menzione speciale merita la cittadina di Hispellum. Qui i convenuti, ascoltato nella basilica di S. Maria maggiore un eccezionale concerto eseguito su un rinomato organo del 1700 e dopo un ricevimento nel palazzo civico, sono stati accolti, per la degustazione di varie specialità gastronomiche, nei caratteristici locali destinati alla "Sagra della bruschetta", la tradizionale festa che si ripete ogni anno, con grande concorso di turisti, per l'assaggio del profumato prodotto degli olive del monte Subasio: un ambiente prettamente medioevale, che riportava la mente molto indietro nel tempo ed ha lasciato nell'animo di tutti un gradito e duraturo ricordo. Il Seminario é stato oggetto di estesi resoconti della grande stampa nazionale ed di una ripresa televisiva andata in onda nelle edizioni del telegiornale di martedi 12 ottobre. 